\title{
Link optimisation for DWDM transmission with an optical phase conjugation
}

\author{
Paweł Rosa*, Giuseppe Rizzelli, and Juan Diego Ania-Castañón \\ Instituto de Óptica, Consejo Superior de Investigaciones Cientificas, Madrid 28006, Spain \\ *p.g.rosa@icloud.com
}

\begin{abstract}
:
We numerically optimise the span length that gives the lowest signal power asymmetry between transmitted and conjugated channels in a DWDM transmission with fibre based mid-link optical phase conjugation.
\end{abstract}

(C) 2016 Optical Society of America

OCIS codes: (060.1660) Coherent communications; (060.2320) Fibre optics amplifiers and oscillators; (060.4370) Nonlinear optics, fibers.

\section{References and links}

1. P. P. Mitra and J. B. Stark, "Nonlinear limits to the information capacity of optical fibre communications," Nature, 411, 1027-1030 (2001).

2. A. D. Ellis, J. Zhao, and D. Cotter "Approaching the Non-Linear Shannon Limit," J. Lightw. Technol. 28(4), 423-433 (2010).

3. I. R. Gabitov and P. M. Lushnikov, "Nonlinearity management in a dispersion-managed system,” Opt. Lett. 27(2), 113-115 (2002).

4. J. D. Ania-Castañòn, I.O. Nasieva, N. Kurukitkoson, S.K. Turitsyn, C. Borsier, and E. Pincemin, "Nonlinearity management in fiber transmission systems with hybrid amplification," Opt. Commun. 233(4-6), 353-357 (2004).

5. Arthur James Lowery, "Fiber nonlinearity pre- and post-compensation for long-haul optical links using OFDM," Opt. Express 15(20), 12965-12970 (2007).

6. E. Ip and J. M. Kahn, "Compensation of dispersion and nonlinear impairments using digital backpropagation," J. Lightw. Technol., 26(20), 3416-3425 (2008).

7. D. Rafique, J. Zhao, and A. D. Ellis, "Digital back-propagation for spectrally efficient WDM 112 Gbit/s PM m-ary QAM transmission," Opt. Exp., 19(6), 5219-5224 (2011).

8. E. Temprana, E. Myslivets, B.P.-P. Kuo, L. Liu, V. Ataie, N. Alic, S. Radic, "Overcoming Kerr-induced capacity limit in optical fiber transmission," Science 26, 348(6242), 1445-1448 (2015).

9. S. L. Jansen, D. van den Borne, G. D. Khoe, H. de Waardt, P. M. Krummrich, and S. Spalter "Phase conjugation for increased system robustness" in Optical Fiber Communication Conference and Exposition and The National Fiber Optic Engineers Conference, Technical Digest (CD) (Optical Society of America, 2006), paper OTuK3.

10. M. D. Pelusi and B. J. Eggleton "Optically tunable compensation of nonlinear signal distortion in optical fiber by end-span optical phase conjugation," Opt. Express 20(7), 8015-8023 (2012).

11. I. D. Phillips, M. Tan, M.F.C. Stephens, M. McCarthy, E. Giacoumidis, S. Sygletos, P. Rosa, S. Fabbri, S. T. Le, T. Kanesan, S. K. Turitsyn, N. J. Doran, and A. D. Ellis "Exceeding the nonlinear Shannon limit using Raman fibre based amplification and optical phase conjugation," in Optical Fiber Communication Conference, OSA Technical Digest (online) (Optical Society of America, 2014), paper M3C.1.

12. M. Tan, P. Rosa, I. D. Phillips, and P. Harper "Extended Reach of 116 Gb/s DP-QPSK Transmission using Random DFB Fiber Laser Based Raman Amplification and Bidirectional Second-order Pumping," in Optical Fiber Communication Conference, OSA Technical Digest (online) (Optical Society of America, 2015), paper W4E.1.

13. P. Rosa, G. Rizzelli, M. Tan, P. Harper, and J. D. Ania-Castañòn, "Characterisation of random DFB Raman laser amplifier for WDM transmission," Opt. Express 23(22), 28634-28639 (2015).

14. M. Tan, P. Rosa, S. T. Le, Md. A. Iqbal, I. D. Phillips, and P. Harper "Transmission performance improvement using random DFB laser based Raman amplification and bidirectional second-order pumping." Opt. Express 24(3), 2215-2221 (2016). 
15. P. Rosa, G. Rizzelli, and J. D. Ania-Castañón "Signal power symmetry optimization for optical phase conjugation using Raman amplification," in Proceedings of Nonlinear Optics, OSA Technical Digest (online) (Optical Society of America, 2015), paper NW4A.36.

16. P. Rosa, S. T. Le, G. Rizzelli, M. Tan, and J. D. Ania-Castañón "Signal power asymmetry optimisation for optical phase conjugation using Raman amplification," Opt. Express 23(25), 31772-31778 (2015).

17. S. K. Turitsyn, S. A. Babin, A. E. El-Taher, P. Harper, D. V. Churkin, S. I. Kablukov, J. D. Ania-Castañón, V. Karalekas, E. V. Podivilov "Random distributed feedback fibre laser,” Nature Photonics 4, 231-235, (2010).

18. M. Alcon-Camas, A. E. El-Taher, J. D. Ania-Castañón, and P. Harper "Gain Bandwidth Optimisation and Enhancement in Ultra-long Raman Fibre Laser based Amplifiers," in European Conference and Exhibition on Optical Communication (ECOC), (IEEE, 2010), paper P1.17.

19. M. Tan, P. Rosa, Md. A. Iqbal, I. D. Phillips, J. D. Ania-Castañón and P. Harper "RIN Mitigation in Second Order Pumped Raman Fibre Laser Based Amplification," in Asia Communications and Photonics Conference, (OSA 2015), paper AM2E.6.

20. C. R. S. Fludger, V. Handerek and R. J. Mears "Pump to Signal RIN Transfer in Raman Fiber Amplifiers," J. Light. Tech. 19(8), (2001).

21. M. Tan, P. Rosa, I. D. Phillips, and P. Harper "Long-haul Transmission Performance Evaluation of Ultra-long Raman Fiber Laser Based Amplification Influenced by Second Order Co-pumping," in Asia Communications and Photonics Conference, OSA Technical Digest (online) (Optical Society of America, 2014), paper ATh1E.4.

22. M. Tan, P. Rosa, S. T. Le, I. D. Phillips, and P. Harper "Evaluation of 100G DP-QPSK long-haul transmission performance using second order co-pumped Raman laser based amplification," Opt. Express 23(17), 2218122189 (2015).

23. K. Solis-Trapala, T. Inoue, and S. Namiki "Signal power asymmetry tolerance of an optical phase conjugationbased nonlinear compensation system," in European Conference and Exhibition on Optical Communication (ECOC), (IEEE, 2014), paper We.2.5.4

\section{Introduction}

The nonlinear-Shannon limit sets a cap to maximum capacity in single mode optical fibres [1,2]. Several techniques have been proposed over the years to compensate or partially mitigate fibre nonlinear effects, pre-shaping and in-line nonlinearity management [3-6] to digital compensation through techniques such as back-propagation [6-8]. Amongst these options, mid-link [9] or transmitter-based [10] optical phase conjugation (OPC) has proven to be one of the most promising, enabling real time compensation of all deterministic (signal $\times$ signal) nonlinear impairments. However, the degree of nonlinear compensation using mid-link OPC is related to the symmetry match of the conjugated and transmitted signal power evolution in the fibre. Meaningful performance improvement has only been demonstrated in Raman-based amplification optical links [11], thanks to the better control over signal symmetry provided by distributed amplification, as well as its improved noise performance. The key to maximise performance in OPC-assisted systems lies in reducing signal power asymmetry within the periodic spans while ensuring a low impact of noise and non-deterministic nonlinear impairments in the overall transmission link.

It has been demonstrated that half- open-cavity random distributed feedback (DFB) Raman laser amplifier with bidirectional $2^{\text {nd }}$ order pumping [12-14] can reduce signal power evolution asymmetry inside the span with respect to its middle point and shows the highest level of symmetry achieved up to date $[15,16]$.

In order to investigate the best practical Raman-based link design and the potential impact of the reduced asymmetry between transmitted and conjugated channels, we consider two DWDM grids (original and conjugated) of 20 channels with a $25 \mathrm{GHz}$ spacing (original and conjugated) that are simulated independently across the C-band with frequency range from 192 $195,775 \mathrm{THz}$. We also show the optimised single channel in-span signal power asymmetry variation due to wavelength dependent Raman gain and attenuation at different frequencies and span lengths. 


\section{Amplification setup}

In our search for an optimal setup for WDM transmission with an OPC we consider random DFB Raman fibre laser amplifier [12,13] that shows the best in-span asymmetry performance comparing with other Raman amplification schemes $[15,16]$. The schematic design is shown in Fig. 1.

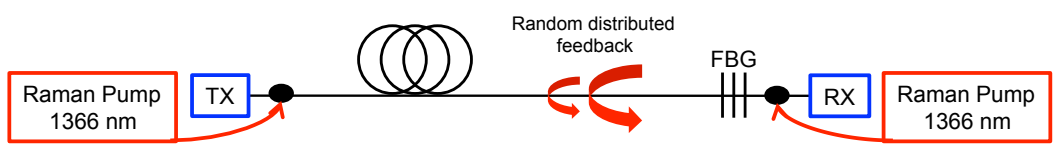

Fig. 1. Schematic design of random DFB Raman laser amplifier.

To form a distributed $2^{\text {nd }}$ order random DFB Raman laser amplifier amplifier, fully depolarised Raman fibre laser pumps are downshifted in wavelength by two Stokes with respect to the frequency of the signal. High reflectivity (99\%) FBG centred at $1455 \mathrm{~nm}$ with a $200 \mathrm{GHz}$ bandwidth was deployed at the end of the transmission line to reflect Stokes-shifted light from the backward pump at $1366 \mathrm{~nm}$ and form a random DFB lasing [17] at the frequency specified by the wavelength of the FBG acting as a first order pump that amplified the signal in the Cband. The advantage of this model is that the gain bandwidth and profile can be modified by selecting appropriate FBG [18] rather than deploying a seed at different wavelength. The lack of an FBG on the side of the forward pump reduces the RIN transfer [19] from the forward pump to the Stokes-shifted light at $1455 \mathrm{~nm}$ at the cost of a reduction in the power efficiency conversion in comparison to the $1^{\text {st }}$ order Raman and URFL amplification schemes. This is particularly important, as forward-pumping RIN transfer from inherently noisy high-power pumps can seriously hinder data transmission [20-22].

\section{Wavelength dependent asymmetry}

To show wavelength dependent in-span asymmetry we simulated single channel across the $30 \mathrm{~nm}$ C-band (1531 - $1561 \mathrm{~nm})$ with a $25 \mathrm{GHz}$ step. Our broadband amplification model includes not only cascaded amplification, but takes also into account residual Raman gain from the primary pump at $1366 \mathrm{~nm}$ to the signal in the C-band, pump depletion from both pumps to the lower order pumps and signal components, double Rayleigh scattering and amplified spontaneous emission noise for each of the signals as well as parameters (attenuation curve at different frequencies, Rayleigh backscattering and Raman gain coefficients) for standard SMF28 fibre used in the simulations. The full description can be found in [13]. The span length ranged from $50-70 \mathrm{~km}$ and the pump powers were optimised to give $0 \mathrm{dBm}$ net gain and the lowest in-span asymmetry at each distance.

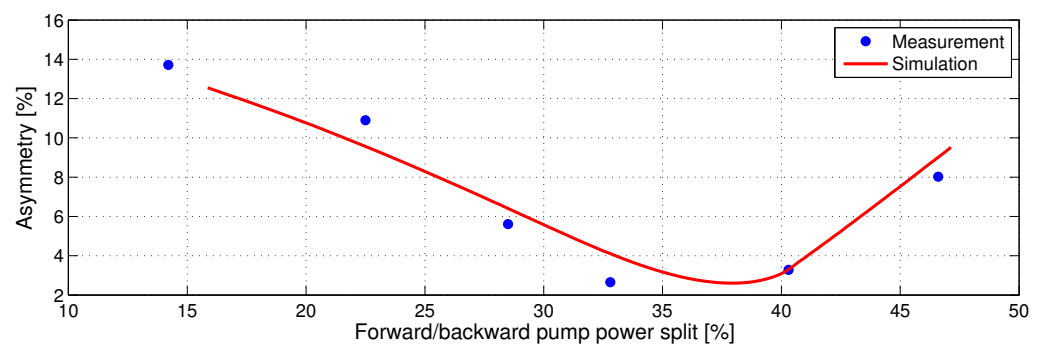

Fig. 2. Asymmetry excursion dependence on the forward and backward pump power split measured at the central wavelength at $1545 \mathrm{~nm}$ in a $60 \mathrm{~km}$ span. 
The forward and backward pump power split for an optimised asymmetry within the span can vary with distance. In Fig. 2 we show the experimental measurement with simulated fit of a pump power split measured at the central wavelength at $1545 \mathrm{~nm}$ in a $60 \mathrm{~km}$ span.
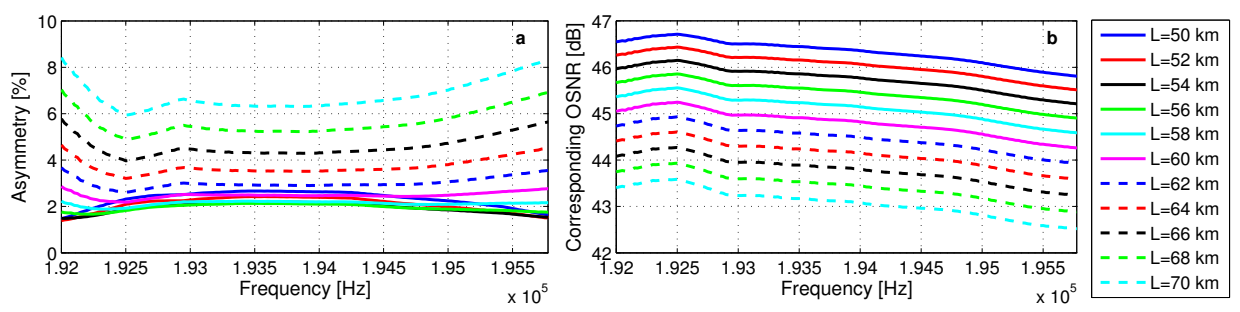

Fig. 3. Signal power asymmetry at given frequency for different span lengths (a) and the corresponding OSNR (b).

The lowest asymmetry (calculated as in [23]) and corresponding OSNR at given frequency for each distance is shown in Fig. 3. With the higher span length the asymmetry variation across the residual grid is more pronounced, hence the optimisation of the link for the wideband DWDM transmission is important as the performance of an OPC is directly related to the symmetry of the transmitted and conjugated channel. The flattest and the lowest overall asymmetry excursion across the simulated band was found at $58 \mathrm{~km}$ (Fig. 4).

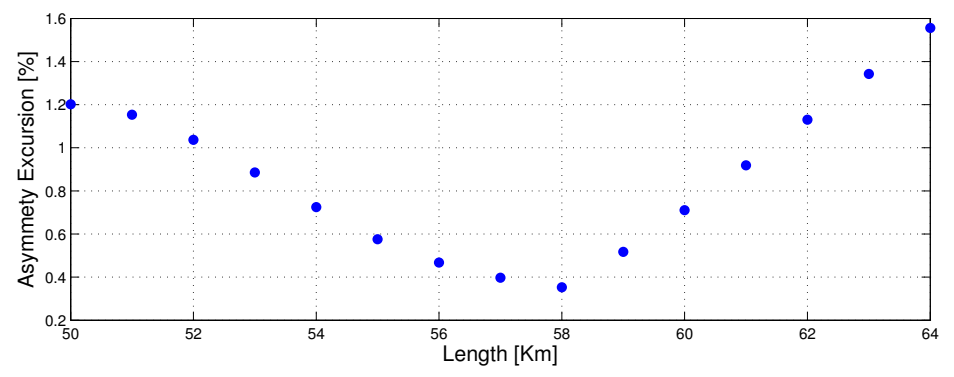

Fig. 4. Asymmetry excursion of a single channel across C band (1531-1561 nm).

The lowest asymmetry as well as asymmetry excursion across the measured band is found to be for the span lengths below $62 \mathrm{~km}$ (solid curves in Fig. 3[a]), hence further optimisation for WDM transmission will be performed in that region.

\section{DWDM transmission with a mid-link OPC}

In DWDM transmission with a mid-link OPC we independently simulate the power evolution of the original channels and their conjugated copies, that is shifted in frequency. The channel count was set to 20 , with a $25 \mathrm{GHz}$ spacing. We assumed $300 \mathrm{GHz}$ spacing for optical phase conjugator. The grid was then being downshifted in wavelength by $500 \mathrm{GHz}$ until the $30 \mathrm{~nm}$ band (1531 - $1561 \mathrm{~nm}$ ) was fully covered. A diagram depicting the simulated frequency sections is shown in Fig. 5.

The asymmetry between transmitted and conjugated channels (inter-span asymmetry) was calculated using the formula:

$$
\text { Asymmetry }=\frac{\int_{0}^{L}\left|P_{1}(z)-P_{2}(L-z)\right| d z}{\int_{0}^{L} P_{1}(z)} \times 100
$$




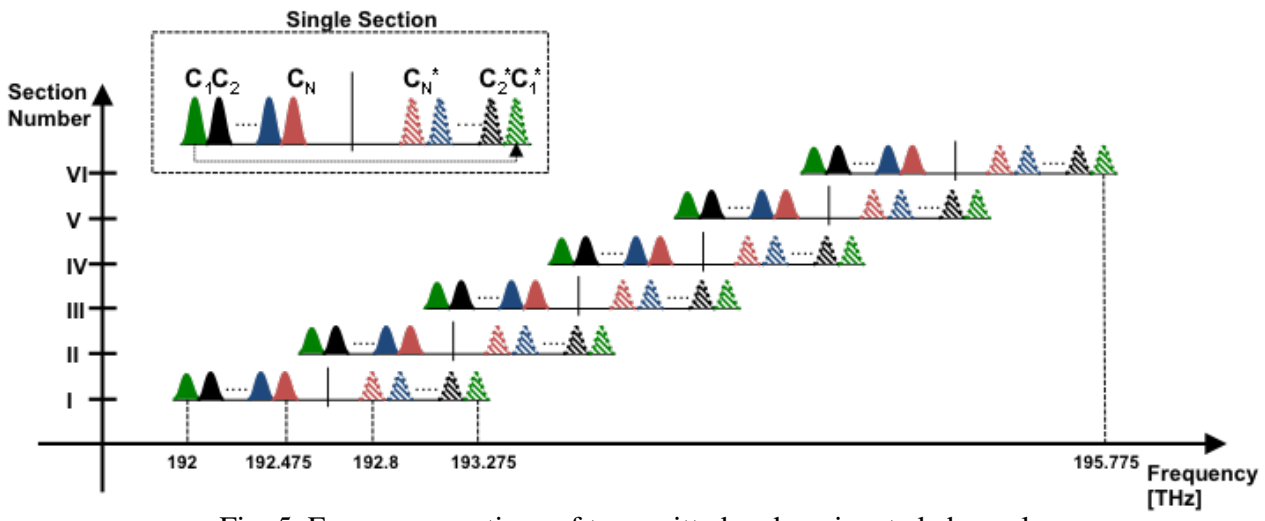

Fig. 5. Frequency sections of transmitted and conjugated channels.

where $L$ is the span length, $P_{1}$ and $P_{2}$ represents average signal power evolution of the transmitted and conjugated channels, respectively.
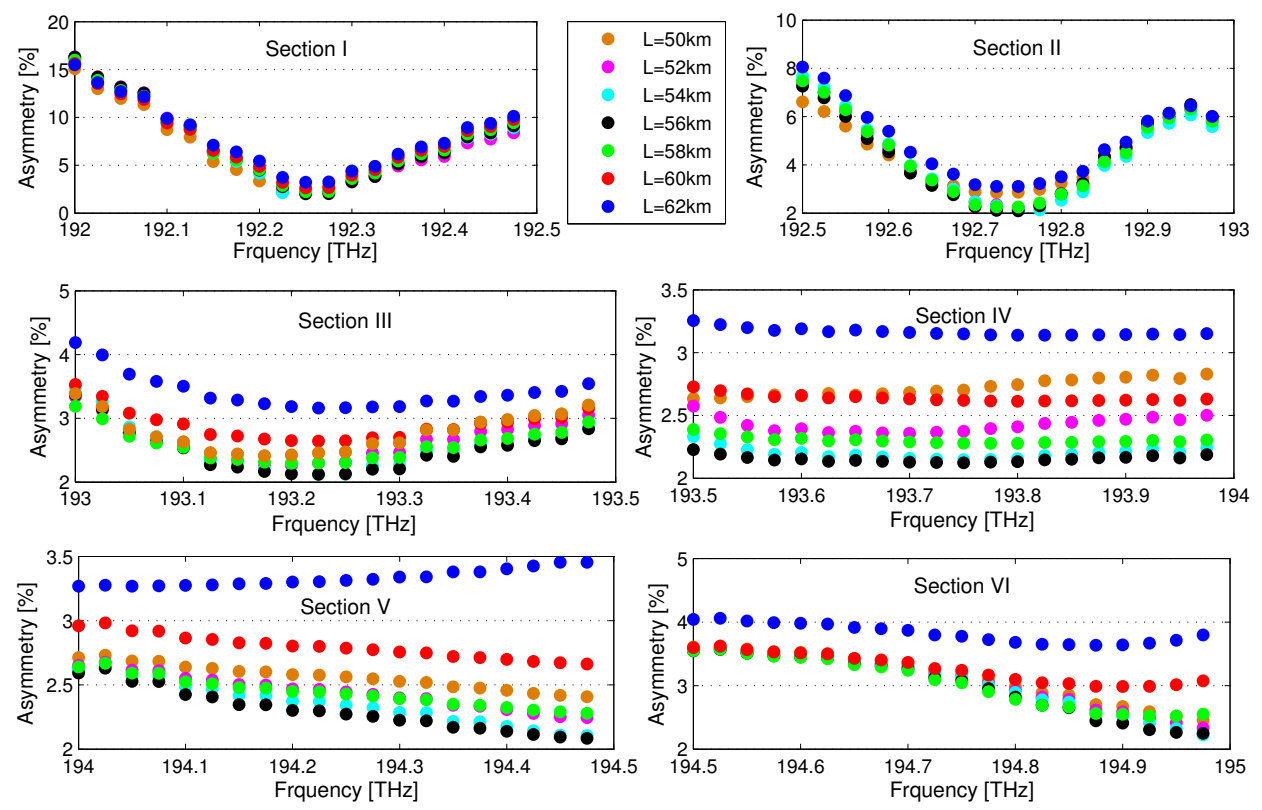

Fig. 6. Optimised asymmetry between transmitted and conjugated WDM channels at different frequency sections. The $\mathrm{Y}$ axes refers to frequencies of the transmitted WDM grid.

Each section of the band was optimised to the channel that gave the best overall asymmetry performance: the grid was simulated to give $0 \mathrm{~dB}$ net gain for the first channel, then the rest of the channels were simulated with the same pump power, next we optimised the grid to a second channel and so on. The same logic was applied to the conjugated copy and finally we compared the asymmetry between original and conjugated channels with all possible combinations. The optimised results with the lowest achievable asymmetry in each section for the distances from 50 to $62 \mathrm{~km}$ links is shown in Fig. 6. Due to the frequency dependence of the attenuation and Raman gain coefficient profiles, the asymmetry in the residual windows (I and II) is most 
pronounced. This is also valid for single channel in-span asymmetry as shown in Fig. 3(a). As a result, the symmetry between transmitted and conjugated channels is greatest for the sections with the best in-span symmetry. Asymmetries below $4 \%$ are found to be achievable for all frequency sections from $193-195.775 \mathrm{THz}$ (window III, IV, V and VI) at all span lengths considered.

Comparing the results from Fig. 6 we can notice the importance of span length optimisation for wide band WDM transmission with an OPC. A span length difference of only $4 \mathrm{~km}$ can lead to a strong performance decrease in nonlinear compensation using OPC due to the associated increase in asymmetry.

\section{Conclusion}

We have evaluated, for the first time, signal power asymmetry between transmitted and conjugated channels in a WDM transmission in Raman-amplified systems with mid-link OPC. We have shown that for the chosen typical fibre-based OPC characteristics and a 20-channel, $25 \mathrm{GHz}$-spaced grid, a $56 \mathrm{~km}$ span length provides most suitable solution that gives the best asymmetry performance, with values below $3 \%$ across most of the C-band. In terms of optimal channel location, the spectral window starting in $193.5 \mathrm{THz}$ (window IV) offers the best possible performance for all span lengths studied.

\section{Acknowledgement}

We acknowledge the support of the EU through the Marie Skłodowska-Curie IF CHAOS for P. Rosa (658982) and FP7 ITN programme ICONE (608099), Spanish MINECO grant ANOMALOS (TEC2015-71127-C2) and Comunidad de Madrid grant SINFOTON (S2013/MIT-2790-SINFOTON-CM). 